\title{
EXPERIMENTAL STUDY OF THE THERMAL PERFORMANCE BEHAVIOR OF ELECTRIC POWER TRANSFORMERS
}

\author{
Nawras Mohammad Azbar ${ }^{1}$
}

\author{
${ }^{*}$ Hayder Mohammad Jaffal ${ }^{2}$
}

1) MSc /Mechanical Engineering Dept., University of Mustansiriyah Baghdad-Iraq.

2) Asst. Prof Dr. Mechanical Engineering Dept., University of Mustansiriyah Baghdad-Iraq.

\begin{abstract}
Electric power transformers of various capacities are one of the most important units of the electrical system. They are considered to be economically costly and have negatively impacted the event of their breakdown. Most electrical transformers fail to perform when the temperature rises and it is mostly requested at its peak. Therefore, it needs periodic maintenance to prevent it from collapsing. Experimentally, distribution transformers (250 kVA, 11/0.416 kV, Core type, Oil engrossed, and Natural convection) have been collected and provided with several sensors to carry out required tests under different operating conditions. All tests were done at AL-Wazyria Company, Iraq for electrical industries. Analyzing the thermal performance of the electrical transformer is related to choosing the appropriate and effective method for cooling the transformer. From the results, it was observed that the oil temperature of the electrical transformer is affected by the temperature of the ambient and the windings, to varying degrees. Accordingly, changing the temperature of the air surrounding the fin has a significant effect on the oil temperature compared to the temperature change of the coils. Therefore, any method that targets the external shape of the transformer and the fins may give the best thermal performance with respect to the improvements that target the coils.
\end{abstract}

Keywords: Power transformer, Experimental study, thermal performance, Methodology, Natural convection.

\section{Introduction}

In general, we need a number of electrical transformers to transfer electrical current from distribution stations to the consumer. Losses in the active parts of the transformer occur during the passage of electrical current leading to a decrease in their efficiency. The conversion efficiency may reach about $93 \%$ in the best conditions, which means there are significant losses in the energy produced. The most important factors affecting the transformer efficiency are temperature, humidity, overload, and type/quality of coil insulation. Any study that provides an improvement to its transformer efficiency, even a slight one, helps to increase the life of the transformer and reduce its maintenance. Many researchers have studied the mechanism of improving the performance of the electrical transformer and increasing its efficiency. Ashish and Animesh 2017 [1] Performed a numerical study to improve the thermal performance of the electrical transformer that transmits heat by natural load. A dynamic computational fluid analysis (CFD) 
was performed on three geometrical shapes represented by rectangular, trapezoidal, and triangular. The results indicated that the best thermal performance of the transformer was while using a trigonometric fin. S. Lee et al. [2] conducted an analytical and numerical study of an electric transformer type (ONAN) to verify its thermal and structural performance. The effect of oil expansion, inside the transformer, was examined during the rise in temperature for the different loads that generate internal pressure on the internal transformer parts. A corrugated fin design has been proposed to avoid variations in internal oil pressure. Wavy fin design meets thermal and structural safety requirements in working condition. Farhan et al. 2019 [3] Suggested different fins shape to study the optimum shape to surpass the heat transfer characteristics to be used in transformers cooling. Pressure drop is supreme in rectangular fins and it declines sharply for lower (h/w) ratio especially at the bends. It was detected that a converse proportionality of temperature with the flow due to the phenomenon of heat transfer. It was note that smooth temperature drops for conical shaped fins. Nasir at al. 2019 [4] Reviewed previous studies on ways to improve the thermal performance of the electrical transformer. It was found that the aluminum fin provided the best thermal performance for the transformer. Increasing the distance between the fins and adding obstacles also improves efficiency. Finned coating improves the heat transfer process. Carrying cracks and openings within the fin body increases the coefficient of heat transfer. Dušan et al. 2015 [5] Studied the increasing of lifetime of the power transformer by explaining the thermal field using of ANSYS (CFX). Also, using of several forms of insulating oil and cooling method. It was concluded that synthetic and natural oils are more suitable than mineral oils of low viscosity. This is due to the small value of the heat flow rate of the oil in the natural cooling. Sean. 2018 [6] presented a three-dimensional numerical simulation to study the thermal performance of cylindrical transformers. Dealt with the effect of the fins on the change in the oil density. Initially, it was conducted a numerical analysis of the transducer without the presence of side fins. After that, was simulated adding the fins to the transducer and studied the effect of adding nails to the surface of the fin and the base. The results show that adding spikes to both the fin and base increases the heat transfer, but adding spikes to the base alone does not guarantee an increase in heat transfer. Arun and Manvendra 2016 [7] used Natural oils in place of mineral oils such as coconut oil, palm oil, corn oil and sunflower oil for cooling and insulation for the electrical transformer. The coolant fin model was designed using (UG 8.0) and analyzed in CFD (Flow Vision). The analysis results showed that the temperature decreased by $2.3 \%$ when using corn oil compared to mineral oil. Panitan and Chainarong 2014 [8] Analyzed the thermal properties and nature of the oil movement within the transformer. A numerical model was established to study the methodology of distributing temperatures inside the transformer. This depends on the change that occurs to the density of the oil and oil movement inside the transformer. The results showed that the oil temperature at the top of the transformer is interchangeably down to the bottom, so it is recommended to increase the surface area of the fin at the top of the transformer. Mushtaq 2017 [9] studied the thermal performance of the electrical transformer with the ambient temperature change and the use of nanomaterials added to the transformer oil. A $250 \mathrm{kV}$ distribution transformer was chosen as a study model. Four types of solid particles $(\mathrm{Cu}, \mathrm{Al} 2 \mathrm{O} 3, \mathrm{TiO} 2$, and $\mathrm{SiC})$ were used to form nanofluids with volumetric parts $(1 \%, 3 \%, 5 \%, 7 \%$ and $9 \%)$. 
The results showed that the use of nanomaterials improves the thermal performance of the transformer and gives SiC-Oil nanofluid a lower temperature to the transformer. Ibtisam et al. 2016 [10] Examined a proposed thermal model for a distribution power transformer. A 2D simulation analysis for three-phase transformer (250 KVA 11/.416 KV core type, mineral oil) using ANSYS software to test the effect of nanoparticles on heat transfer process. The insulation oil was different with Nanofluid, two types of Nano particles ( $\mathrm{CuO}$ and $\mathrm{Al} 2 \mathrm{O} 3)$ with $(0.5 \%)$ as a volume concentration was used, where the temperature reduced about (5\%). Changed from silicon steel to amorphous steel for coil material, caused a reduction in temperature about (9.9\%) in high voltage winding. From previous studies, various methods have been observed to improve the thermal performance of electrical transformers. It was noted that there was no extensive study to clarify the methodology of the distribution of temperatures inside the transformer. Its relationship to the surrounding conditions plays an effective role in choosing the appropriate method of improvement for the thermal performance of the transformer. The scope of this work is limited to the methodology for distributing temperatures within the transformer. For experimental work, the distribution transformer used are $(250 \mathrm{kVA}, 11 / 0.416 \mathrm{kV}$, base type, submerged oil, and natural convection) compiled by Al- Waziriya Electrical Industries Company / Iraq. A 250 $\mathrm{kVA}$ transformer was selected as a case study, which is widely used in the Iraqi electricity grid.

\section{Experimental Work}

The experimental aspect is shown in this paragraph. The distribution transformer used are (250 kVA, 11 / $0.416 \mathrm{kV}$, base type, oil immersed, and natural thermal load) assembled at the State Company for Electrical Industries in
Al-Waziriya / Iraq. A $250 \mathrm{kVA}$ transformer was chosen as a case study, which is widely used in the Iraqi electricity grid. Figure (1) shows an image of this converter. The transformer consists of three copper coils and a steel core connecting them, all coils are immersed in oil, and the transformer body is equipped with fins to increase the heat transfer area. The oil plays two important roles, the first as a cooling medium by transferring the heat generated in the coils out. The second, as an electrical insulator. Dimension of ONAN distribution transformer presented in the table (1).

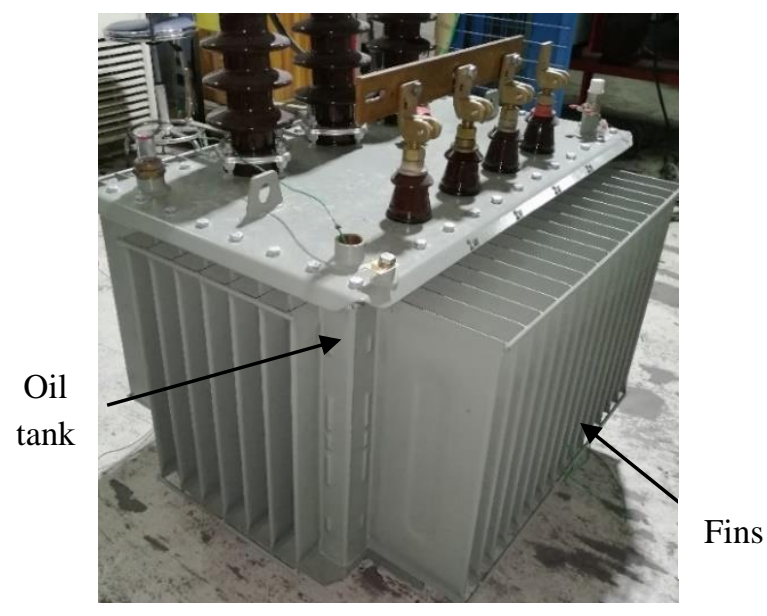

Figure 1. Traditional electrical transformer geometry.

Table 1. Dimension of traditional electrical transformer.

\begin{tabular}{|c|c||}
\hline Descriptions & Dimension \\
\hline \hline Casing height & $0.7 \mathrm{~m}$ \\
\hline \hline Casing length & $0.9 \mathrm{~m}$ \\
\hline \hline Casing width & $0.45 \mathrm{~m}$ \\
\hline \hline Fin height & $0.6 \mathrm{~m}$ \\
\hline \hline Fin Length & $0.2 \mathrm{~m}, 0.08 \mathrm{~m}$ \\
\hline \hline Number (fins) & 50 \\
\hline \hline Space between & $0.04 \mathrm{~m}$ \\
\hline \hline Total fins area & $9.6 \mathrm{~m}^{2}$ \\
\hline \hline Total & $12.3 \mathrm{~m}^{2}$ \\
\hline \hline
\end{tabular}




\subsection{Technical specifications for electrical} transformer

The experimental aspect includes assembling the transformer at the abovementioned site for the purpose of preparing it for examinations within the company's own laboratories. The transformer frame is made of high-quality silicon iron which has been cut to dimensions according to the design required for the transformer capacity. The electrical coils are made of copper and the insulation material used in the transformer coil is an insulating coil containing the diamond squares coated on both sides. The insulation material is compressed between the coils and dried by the drying machine shown in Figure (2). The drying machine makes the coils solid and strong as one unit. The core of the transformer is placed inside a tank surrounded by hollow fins, so that the oil passes through it to ensure more heat exchange to cool the transformer. Transformer tank with hollow fins is shown in Figure (3).

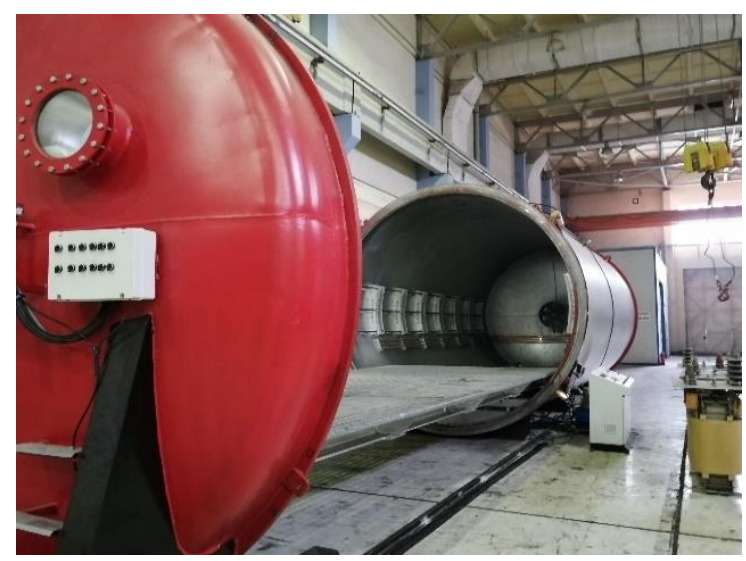

Figure 2. Drying machine.

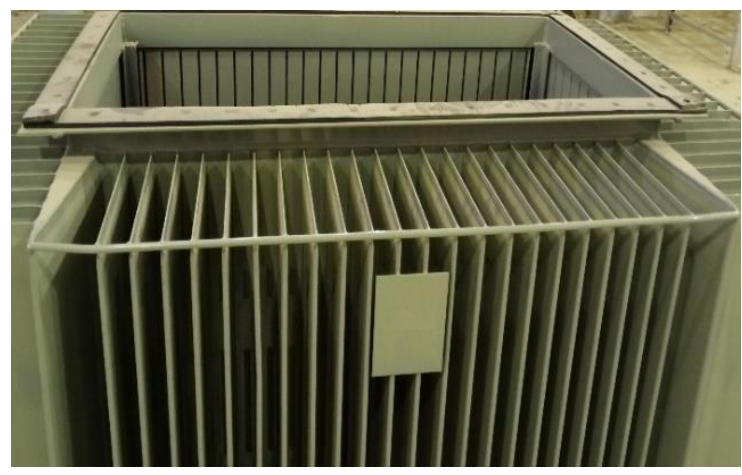

Figure 3. Transformer tank with hollow fins.
Distribution transformers are manufactured inside the factory according to specific specifications, where the operations are done as follows:

1- Silicon iron is cut with specific dimensions, after which the piece is passed with a special mold for the purpose of forming the body of the external transformer represented by the tank and hollow fins.

2- The thin copper layers are created for the purpose of wrapping them, with the addition of a layer of chemically untreated paper buffer, for the purpose of enabling the paper to be saturated with oil to the maximum degree possible.

3- The files are compressed and dried by a special machine and drying ovens.

4- All copper connections, as well as nonvoltage transformer and ceramic insulators are also assembled.

5- Any moisture is removed from the coils and the transformer body is dried before the parts are placed in the tank.

6- The tanks are cleaned with chemicals and the transformer body is coated with three layers to protect the tanks from rust and weather.

7- The active parts of the transformers are grouped inside the tanks and then filled with oil. Transformer oil properties: density $(870$ $\left.k_{g} / m^{3}\right)$, Specific heat $\left(2000 \mathrm{~J} / k_{g} \cdot \mathrm{k}\right)$, thermal conductivity $(0.109 \mathrm{~W} / \mathrm{m} . \mathrm{K})$, Viscosity $\left(0.0124 k_{g} / \mathrm{m} . \mathrm{s}\right)$.

8- After the transformer assembly is completed, all routine tests are performed according to international standards (IEC60076).

\subsection{Tests of Transformer}

2.2.1 Test to measure the losses of Electrical Transformer 
After completing the assembly of the transformer, the transformer is transferred to the aforementioned company laboratories for the purpose of starting electrical and thermal tests.

1- H.V. and L.V. coil resistances are measured to compare the same corresponding values in the datasheet of design.

2- Transformation ratio is measured by connecting one phase of the H.V. coil to the same phase in the L.V. coil.

3- In this step, the dielectric between the components of the transformer is measured, to make sure that the tank of transformer, coils, and core are isolated.

4- Losses at no load were measured by supplying voltage to L.V. side by using power analyzer (PM300), while H.V. side is opened.

5- Load losses were critically measured as the final step in the test. Voltage is supplied to H.V. side and increasing it until the line current is reached. L.V coil is shorted and, the load losses are measured by power analyzer (PM300).

\subsubsection{Thermal Test of Transformer}

After assembling the electrical transformer and conducting all laboratory tests within paragraph (2.3.1) and its success. The transducer is transferred to a special laboratory to load loads on the coils and monitor the oil temperature over time. The electrical transformer shall be prepared for thermal examination according to the following steps:

1- Making (short circuit) columns of transformer coil for (LV) and connecting (HV) with wires that provide the voltage determined by the examiner, Knowing that the amount of applied voltage is related to the high temperature of the electrical coils.
2- Providing the transformer with a set of eight thermocouple devices distributed as follows:

- Four thermocouple sensors are installed on both sides of the tank facing the (L.V) and (H.V) at the top and bottom.

- One thermocouple sensor placed inside the tank to measure oil temperature.

- Three thermocouple sensors with dispensers with jugs containing an amount of oil placed one meter from the sides of the transformer to measure the ambient temperature.

After completing the above two steps, a test is carried out according to (IEC60076). By setting a specified amount of voltages and we started recording the temperature sensor readings manually for each one-hour operation until the oil temperature reaches stability. Provided that the operating hours are not less than seven consecutive hours. Figure (4) shows the practical aspect.

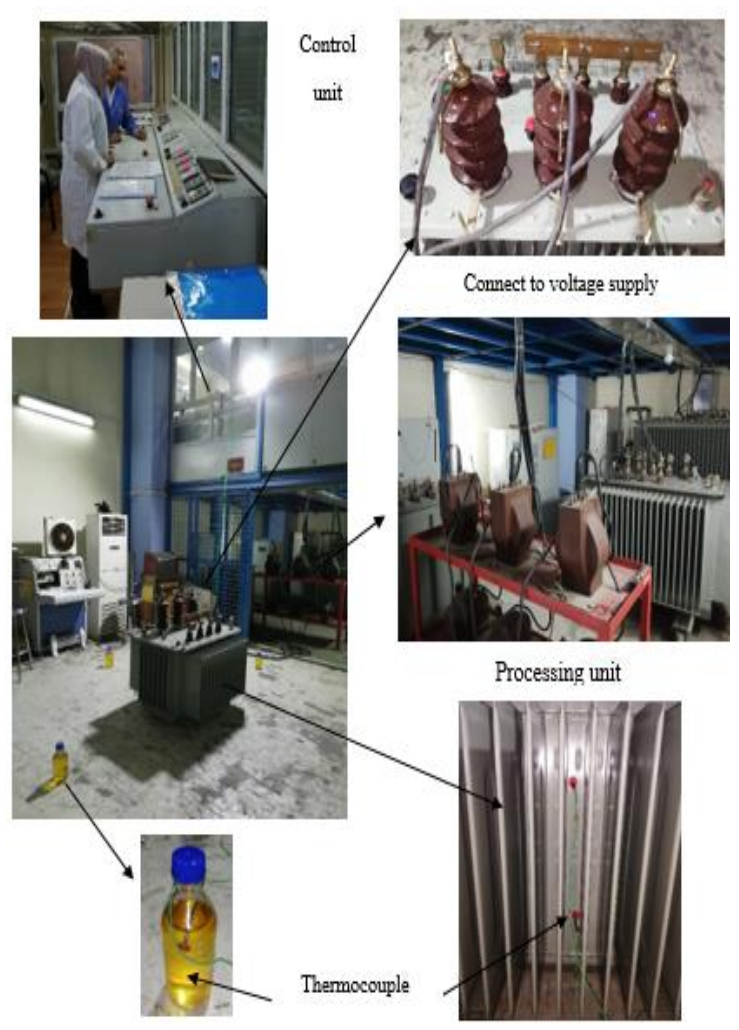

Figure 4. Actual connection of thermal test. 


\section{Measuring Instruments [11]}

- Winding resistance meter type WRM $-10 \mathrm{p}$.

- High voltage insulation (Hi Tester 3455).

- Power analyzer PM300. accuracy (0.1\%).

- Digital Temperature Logger (600 Series).

- Thermocouple type $\mathrm{k}$ rang $(-200$ to +1370$)$ ${ }^{\circ} \mathrm{C}$. accuracy $\left(0.1 \%+0.5^{\circ} \mathrm{C}\right)$.

- The transformer turns ratio tester (ATRT01B S3).

\section{Governing equation}

The value of the heat transfer coefficients is largely dependent on the mean ocean temperatures and the oil coming into contact with the fins. The heat transfer coefficient (h) was determined from standard experimental formulas of natural convection on a vertical plate [12][13].

$p_{r}=\frac{c_{p} \mu}{k}$

$G_{r}=\frac{L^{3} \cdot \rho^{2} \cdot g \cdot \Delta T \cdot \beta}{\mu^{2}}$

$R_{a=} p_{r} G_{r}$

$N_{u}{ }^{1 / 2}=0.82+\frac{0.38 R_{a}{ }^{1 / 6}}{\left[1+\left(\frac{0.49}{p_{r}}\right)^{\frac{9}{16}}\right]^{8 / 27}}$

$h=\frac{N_{u} k}{L}$

where:

(L) length of the fins, (k) thermal conductivity, $(\mu)$ dynamic viscosity, (g) gravitational acceleration, (cp) specific heat, ( $\beta$ ) thermal expansion coefficient.

\section{Test procedure}

After preparing the electrical transformer, the tests related to the current study were started, and included temperature tests for the atmosphere (308 K and $283 \mathrm{~K}$, i.e) making the electrical transformer in winter and summer time for Iraqi airspace. After determining the air temperature in the laboratory (for example, winter), a specific value is dropped from the voltages to the transformer. We started recording the temperature sensors for you for an hour of time until the temperature of the oil has stabilized, provided that the number of test hours is not less than seven consecutive hours. The next day, the experiment is repeated at the same voltage to check the recorded readings. The recheck is done by assigning another value to the voltage at the same selected air temperature and the test is done in the same manner. Repeated the process by projecting five voltages with the same ambient temperature to obtain an average oil stable temperature with the transformer temperature. All the mentioned method of examination is returned, but with a temperature of the ocean (summer) and with the same voltage values that he worked on with the first examination. Then we study the effect of the ambient temperature change by establishing the temperature of the transformer coil and using the same measurement method above.

\section{Results and Discussion}

In this section, the most important experimental results will be analyzed and discussed.

The tests for the traditional electrical transformer were carried out in the laboratories of the General Company for Electrical and Electronic Industries in Baghdad / Al-Waziriya, affiliated to the Ministry of Industry. After preparing the electrical transformer, the tests 
related to the current study were started, and included the temperature tests for the atmosphere $(308 \mathrm{~K})$ and $(283 \mathrm{~K})$. After selecting the ambient temperature in the laboratory (for example, winter), a specific value is thrown from the voltages to the transformer, and we started recording the temperature sensors readings for each hour of time until the temperature of the oil has stabilized (provided that the number of test hours is not less than seven consecutive hours). The next day, the experiment is repeated at the same voltages to ensure the recorded readings are accurate. The recheck is done by assigning another value to the voltage at the same selected air temperature and the test is done in the same manner. The process is repeated by subjected five values of voltages with the same ambient temperature to obtain a stable average oil temperature with the transformer temperature. Then we studied the effect of the ambient temperature change by establishing the temperature of the transformer coil and using the same measurement method above.

Figure (5) indicates the variation of average oil temperature with the time measured in Iraq's weather conditions in the summer, which is 308 $\mathrm{K}$, for the different values the windings temperature of $(330,340,350,360,370 \mathrm{~K})$. From all winding's temperature, it was observed that the average temperature of the oil increases sharply at the beginning of the test until the temperature of the oil reaches stability with time and this degree is considered the stable temperature of the electrical transformer. During the first hours of the test, it was observed that the average oil temperature rises sharply and the number of rises depends on the amount of winding temperature, with the ambient temperature established so that the higher winding temperature is more marginal compared to the low coil temperature.
Figure (6) indicates the variation in the average oil temperature with the time measured in Iraq's weather conditions in the winter, amounting to $283 \mathrm{~K}$, for the different values the windings temperature of $(330,340,350,360,370 \mathrm{~K})$. From the figures, we noticed the same behavior used for the heat of the oil when the ambient temperature is high.

Figure (7) shows the relationship between the average oil temperature of the electrical transformer (in steady state conditions) and the temperature of the windings under Iraqi weather conditions in the summer and winter seasons. It was observed that the average oil temperature of the transformer is affected by the temperature of the windings and the ambient temperature, so they rise in a noticeable linearity for the summer and winter months On the other hand, it was observed that the average temperature of the oil during summer weather conditions is higher than the average temperature of the oil in the winter.

Figure (8) shows the variation in the average oil temperature with time as the coil temperature is stable $(350 \mathrm{~K})$ and the ambient air temperature changes. From this figure, we noted that the average oil temperature increases sharply at the beginning of the test until the oil temperature reaches stability over time. In comparison to Figures (5) and (6), we noticed that the oil temperature of the transformer is affected more when the air temperature changes. As for the temperature change of the windings, their effect on oil temperature is moderate and low, when compared with its effect on air temperature change.

Figure (9) shows the relationship between the average oil temperature at the top and bottom of the transformer with time, where the coil temperature $(350 \mathrm{~K})$ and the air medium (308 $\mathrm{K})$ were determined. From this figure, we 
noticed that the oil temperature in the upper section is much higher than the temperature in the lower section by $9 \%$. This is due to the behavior of the oil due to its different density while gaining temperatures.

Figure (10) shows the variation of the convection heat transfer coefficient with the surface temperature of the electrical transformer after settling within the ambient air temperature $(308,283 \mathrm{~K})$ represented by Iraqi weather conditions in summer and winter. In general, it was observed that the value of the convection heat transfer coefficient between the surface of the transformer and the surrounding decreases gradually as the temperature of the transformer surface increases as a result of the rise in the oil temperature. Meaning that the value of the convection heat transfer coefficient has a higher value as possible at the beginning of the operation of the transformer so that the temperature of the oil and the surface is low to increases the values of the coefficient of heat transfer by convection and gradually decreases with increasing oil temperature and the surface of the transformer. The reason for this is due to the fact that the difference between the surface and surrounding temperature increases, so the values of the convection heat transfer coefficient decreases. It was also noted that the values of the heat transfer coefficient are higher in the summer compared to the winter season, and the reason for that the values of the difference in temperature between the surface and the ambient are large in the winter season, so the values of the of convection heat transfer coefficient decreases.

\section{Conclusions}

Experimental study was conducted to test the effects of operating parameters on the cooling performance of three-phase electrical distribution transformer $(250 \mathrm{kVA}$ oil natural air natural).

1- The average temperature of the oil during weather conditions in the summer is higher than the average temperature of the oil in the winter.

2- Air temperature is the most influencing factor for the transformer average oil temperature relative to the temperature change of coils.

3- The oil temperature in the upper section is much higher than the temperature in the lower section by $9 \%$.

4- The heat transfer coefficient is inversely proportional with the transformer surface temperature. As well as the values of the heat transfer coefficient are higher in the summer compared to the winter season.

From the above-mentioned conclusions, it is possible to understand the methodology of the thermal performance of the transformer, which helps in selecting the appropriate and effective method for cooling the transformer. In summary, we concluded that any improvement to the transformer outer structure (reservoir, fins) may give better thermal performance compared to the improvements related to windings or transformer oil. It is also preferable to target the top of the transformer in the process of improving the thermal performance of the transformer, due to the high oil temperature in this area. Thus, changing the shape of the transformer and the fins is one of the most effective ways to influence the thermal performance of the transformer.

\section{Conflict of interest}

We can prove that there is no any conflict of interest which may arise from the above manuscript. 


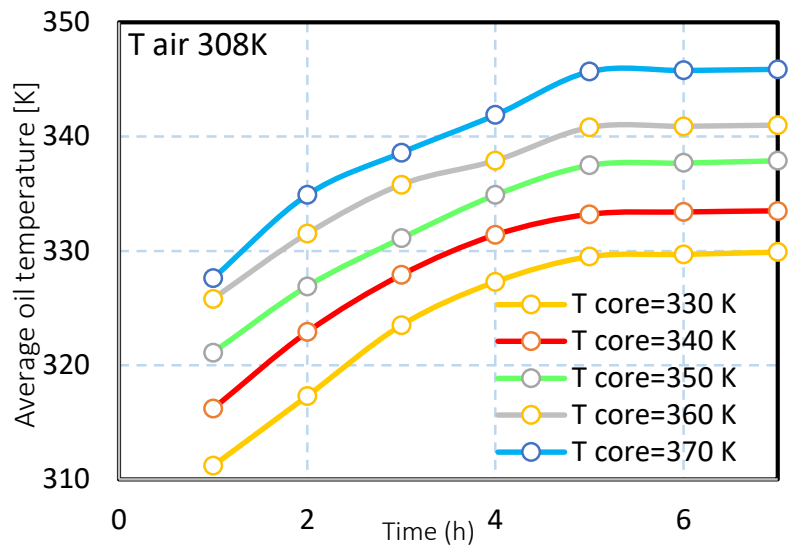

Figure 5. variation the average oil temperature with the time measured in Iraq's weather conditions in the summer.

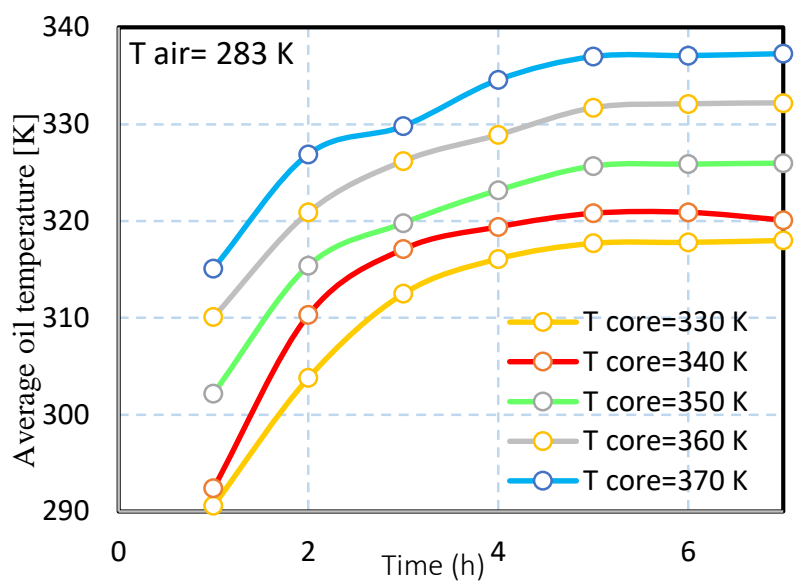

Figure 6. variation the average oil temperature with the time measured in Iraq's weather conditions in the winter.

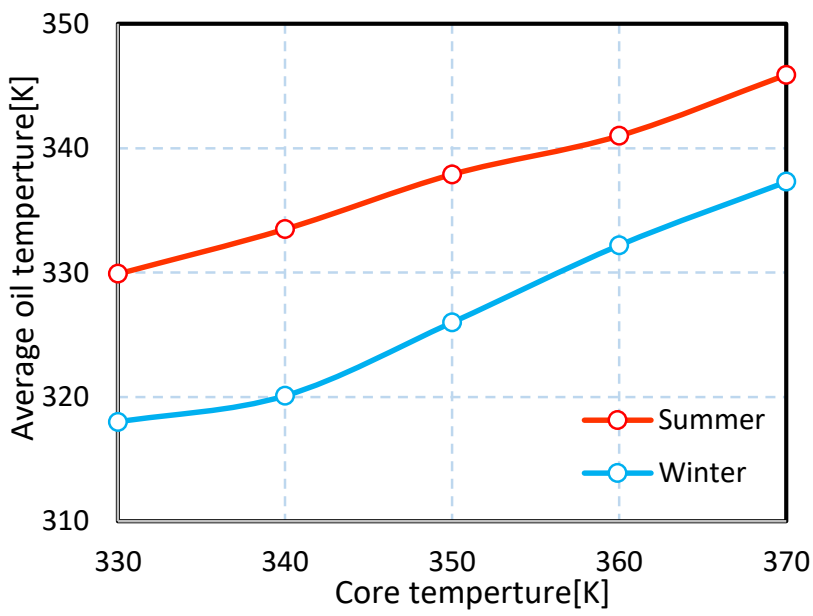

Figure 7. the relationship between the average oil temperature and the temperature of the windings under Iraqi weather conditions.

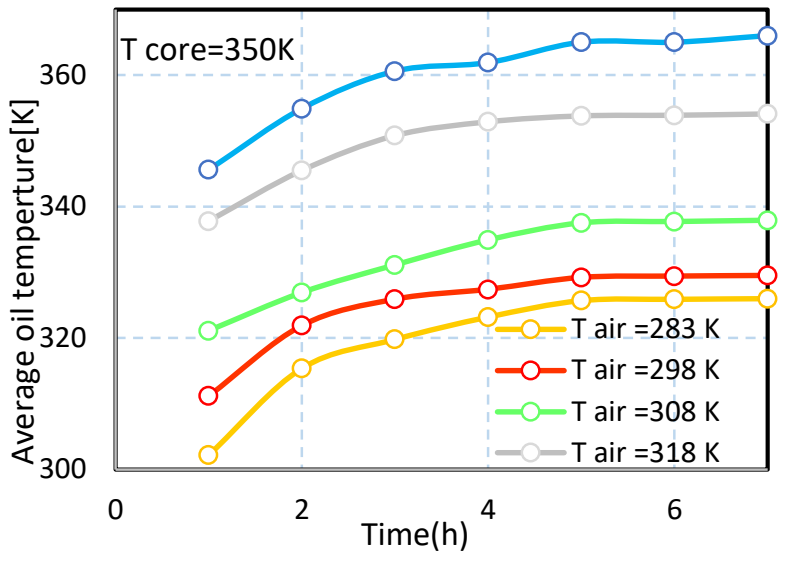

Figure 8. variation the average oil temperature with the time measured in different air temnerature.

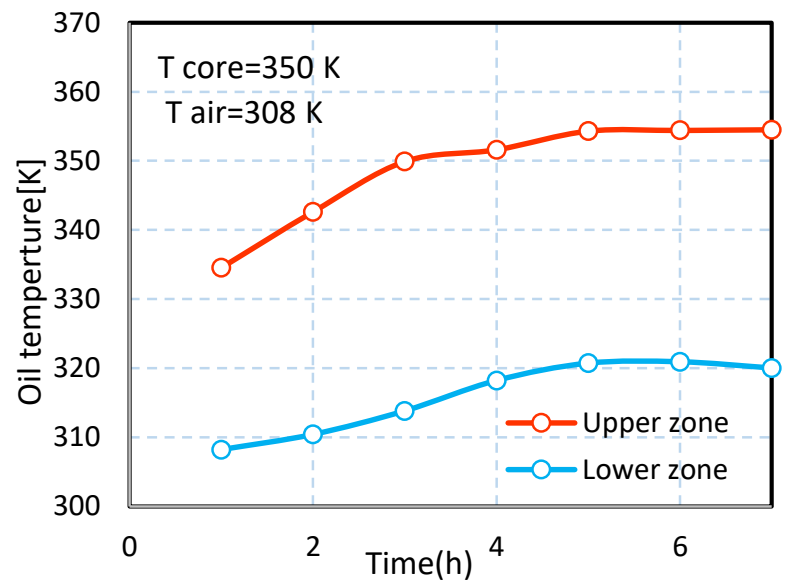

Figure 9. shows the relationship between the average oil temperature at the top and bottom of the transformer with time.

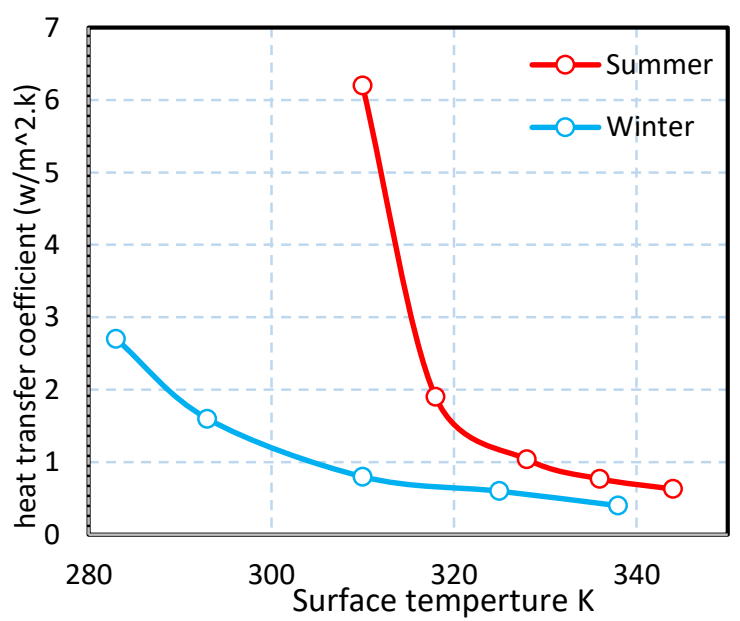

Figure 10. the variation of the convection heat transfer coefficient with the surface temperature of the electrical transformer. 


\section{References}

1. Ashish Davande and Animesh Singhai (2017)" Thermal Analysis of Different Design of Transformer Fins "International journal online of science.

2. S. Lee1, J. Y. Lee1, J. C. Yun1, J. Y. Park1 \& J. H. Woo2(2012)" Development of thermal and structural design technology for a hermetically sealed oil transformer" WIT Transactions on The Built Environment, Vol 126, WIT Pres.

3. Farhan, Saad,Hafiz and Muhammad Anwar (2019) " Heat Transfer Enhancement in Transformers by Optimizing Fin Designs and Using Nanofluids" Arabian Journal for Science and Engineering.

4. Nasir, Bhushan and Nitish (2019) "A Review Paper on Pin Fin Efficiency Enhancement" International Journal of Applied Engineering Research ISSN 0973-4562 Volume 14, Number 9.

5. Dušan, Marek and Ján (2015) "Solution of Thermal Field Around Transformer" international scientific symposium 2015, 1618.9.

6. Sean Ferneyhough (2018) "Numerical analysis of an electrical transformer with fins and horizontal pins" department of mechanical engineering university of nevada, las vegas.

7. Arun N R1and Manavendra (2016) "Performers and Analysis of Transformer Radiator with Different Types of Oils Using CFD" international journal of innovative research in science, engineering and technology vol. 5, issue 12 .

8. Panitan and Chainarong (2014) "Numerical Simulation of Heat Transfer Process in OilImmersed Transformer" the 5th TSME international conference on mechanical engineering 17-19th December 2014, the empress, chiang mai.

9. Mushtaq (2017) "Using the transformer oilbased nanofluid for cooling of power distribution transformer" international journal of energy and environment volume 8 , issue 3 , 2017 pp.229-238.

10. Ibtisam, Sahar R. and Azhar K. (2016)" Theoretical Temperature Distribution Investigation in Electrical Transformer by Using Nano - Technology" Eng. \& Tech.Journal, Vol.34, Part (A), No.12.

11. Aseel A. Ismael (2018) "An Experimental Investigation to Enhance Cooling System of a Distribution Transformer by Using Thermosyphon" thesis of Master of Science in Electromechanical Engineering, the Electromechanical Engineering Department / University of Technology.

12. Robert Sitar, Žarko Janić, Željko Štih. (2015)" Improvement of thermal performance of generator step-up transformers" Applied Thermal Engineering, Vol.78.

13. Mustafa S. Mahdi, Anees A. Khadom, Hameed B. Mahood, Mahmood Abdul Razak Yaqup, Jammal M. Hussain, Khalid I. Salih, Hussein A. Kazem, Effect of fin geometry on natural convection heat transfer in electrical distribution transformer: Numerical study and experimental validation", Thermal Science and Engineering Progress, Vol.14, PP(100414), 2019. 